\title{
AN ARTIFICIAL NEURAL PROCESS TO CREATE CONTINUOUS OBJECT BOUNDARIES IN MEDICAL IMAGE ANALYSIS
}

\author{
Mahinda Pathegama ${ }^{1)}$, Özdemir Göl ${ }^{2)}$ \\ ${ }^{1) 2)}$ School of Electrical and Information Engineering, University of South Australia, \\ Mawson Lakes SA 5095, Australia \\ 1) e-mail: mahinda@iee.org, ${ }^{2)}$ e-mail: ozdemir.gol@unisa.edu.au
}

\begin{abstract}
Computer-aided analysis for cell images acquired by an electron microscope involves a range of image processing steps including edge detection and thresholding. The major problem encountered in automatic cell analysis is the possible presence of incomplete boundaries of cell features, which prevent the generation of cell feature details including all measurements as the boundaries include very tiny gaps. This paper presents a novel edge-linking technique based on an artificial neural process, which uses directional sensitivity derivatives from an edged image. The input signals applied to the neural layer are integrated with direction-sensitive information produced by an auxiliary algorithm, which interrogates all the pixels in the 2-D image in order to designate the specified direction in which each edge-end pixel should propagate. The proposed edge-linking technique, implemented as an image-processing algorithm for direction-sensitive selectiveness, provides an effective solution to the problem of porous boundaries encountered in biological cell image analysis.
\end{abstract}

Keywords: - boundary gaps, edge-linking, edge detection, cell analysis.

\section{INTRODUCTION}

Computer-aided analysis performed on cell images acquired from electron microscopes used in conjunction with sensing modalities is of increasing importance to researchers in the biomedical field because it offers many advantages, especially in the remote analysis of medical images. As computeraided analysis always comprises a series of advanced image-processing steps, a procedure that operates automatically is a pertinent factor for many researchers. Extracted cell boundaries that have a complete perimeter are essential for the generation of a detailed report that has the required final measurements. Cells with incomplete perimeters, even with just one pixel missing, will not be detected and recognised as objects to be analysed. Despite employing the processing steps prevalent in the contemporary practice of image-processing, the existence of even the tiniest of gaps present in the extracted edges impedes the continuation of the automatic processing steps.

This problem of gaps is common to many other types of medical image application. A previous study matching a brain atlas to medical images found that completely connected contours could not be produced despite the application of a number of steps [1]. In their study, although Gaussian filtering, Sobel edge detection, a thinning operation to obtain one-pixel edges and a heuristic search technique to link the edge gaps were all used, open segments remained, which had to be connected manually.

To overcome such difficulties experienced in the automatic or semi-automatic image processing systems, we present here a novel technique for the process of edge-linking which is based on the concept of direction-sensitive cells present in the early stages of the physiology of visual development.

\section{MODELLING THE NEURAL PROCESS}

Neural activities in the human vision system may generate an edge-linkage process according to edge information of reflected images on the retina; this is the premise for the development of the technique described in this paper. Neurons in the visual system do not respond strongly to uniform regions, but rather to luminance discontinuities [2] [3]. Most neurons only respond strongly to edges and do not produce vigorous responses to regions or surfaces [4] [5]. Even under natural viewing conditions, the surface perceived by the vision system depends not only on the light reflected from surfaces but especially on the change in light across the boundaries of the surfaces [6]. If continuous regions carry weak physical signals, the question arises as to how object surfaces can be seen and not only their 
boundaries. Hence, a kind of linkage process by neural responses may be activated to compensate for the immediate lack of physical signals at certain image regions. This neural process appears to be based on signals from direction-sensitive neurons that perceive according to surface directions. The receptive fields may develop internal reactions producing directional sensitivity through their interaction upon such perceptions. This argument leads us to a solution for the edge linkage problem experienced in medical image analysis tasks. Thus, the idea of an edge-linking function, which occurs through the activities of directionally sensitive neurons, has been developed by mimicking the neural processes in the physiology of vision.

\section{TECHNIQUES FOR EDGE - LINKING}

Applying available edge extraction techniques, a binary image with edges of one-pixel in width can easily be obtained for cell image analysis. The analysis of cell images is thus substantially simplified initially by focusing on obtaining information about edges, suspending further pixel information, before proceeding to subsequent steps.

The linkage operates from edge-end pixels on one side of the gap until they meet the next edge-end pixels at the other side.

To complete the edge map, the automatic linkage $\left(z_{i}\right)$ can be represented in 1-D by the following equations:

$$
\begin{aligned}
& \frac{d z_{i}}{d t}=-a z_{i}+b\left(I_{i-1}+I_{i+1}\right)+P+Q \\
& P=\frac{c\left(z_{i-1}-e z_{i}-\theta\right)}{1+g I_{i}} \\
& Q=\frac{c\left(z_{i+1}-e z_{i}-\theta\right)}{1+g I_{i}}
\end{aligned}
$$

where $a, b, c, e, g, \theta$ are the parameters for the neural activations, $\mathrm{z}_{i}$ is the linkage argument and $I_{i}$ the input.

From the above equations, it can be seen that the linkage process of an edge-end pixel is limited to only one linkage connection, while creating new pixel information on the neighbouring pixel positions between the edge pixels which are empty. Iteratively, new pixel information is produced in relation to the corresponding input pixel value $I_{i}$, or until reaching the value 1 in the binary image.

This linking process would appear to closely resemble the behaviour of the visual path and seems to be composed of cellular syncytium. A cellular syncytium is an array of intimately connected neurons such that contiguous neurons can easily pass signals between each other's compartment membranes, possibly via gap junctions [7]. The spreading pattern emanating from the edge-end pixel continues until the gap is closed. The set of pixels thus created is singularly unique and can not be duplicated erroneously.

\section{DIRECTIONAL SENSITIVITY}

The direction-sensitive information of the pixels can be incorporated in the input information of corresponding neighbours in the 1-D equation (1), and the equation becomes:

$$
\frac{d z_{i}}{d t}=-a z_{i}+b\left(\vec{\nabla} I_{i-1}+\bar{\nabla}_{i+1}\right)+P+Q
$$

where $\vec{\nabla}$ and $\bar{\nabla}$ denote the directional sensitivity function. The input $I_{i} \quad(i=1,2, . ., n)$ applied to equations (2) and (3) leads to the output pattern $z_{i}$ $(i=1,2, . ., n)$ of the automatic linkage process via equation (4).

The input accompanied by direction-sensitive information is produced by another algorithm, which searches all the pixels in the 2-D image in order to designate the specified direction in which each edgeend pixel should move as in Fig. 1. This search locates each edge-end pixel and sets a new direction for the pixel. The specified direction for each of the detected pixels takes into account the known alignment of neighbouring pixels, setting the direction of the incomplete pixel to be the opposite to that of the neighbouring cell direction. This directional information passes on to the neural activities given in equation (4) while corresponding pixel values act as the input signal for equation (2) and (3). The pixel values with this directional information are the input signals for equation (4). This direction-sensitive algorithm facilitates the automatic filling of small gaps in order to complete the object boundaries.

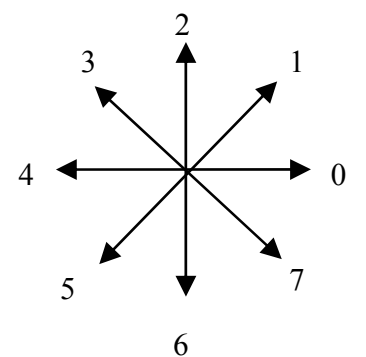

Fig.1 - The eight possible directions for the detected pixels.

The direction-sensitive neurons interacting between themselves are sensitive to opposite directions. The activation consisting of direction selectivity 
organises relevant edge pixel detection, and this results in interaction through neighbouring neurons. This similarity- and proximity-based selection process serves as an internal representation of the linkage process. The edge-end pixel spreads until it achieves edge-end pixel correspondence according to the directional selectivity indicated by directionsensitive neurons.

The directional sensitivity function, which has to accompany the function of equation (4), can be implemented in ways other than the proposed searching algorithm above. One such method could be based on the Chain Coding algorithm by extracting some of its features [8] [9] [10]. Chain Coding is the process of tracing a pixel-wide line starting at an end pixel, finding the next pixel from directional information, and continuing in this way until the last pixel in the line is found. Chain Coding locates end points and uses a single number to describe the direction as shown in Fig. 1.

The function of contour interaction studied in a previous project [11], which used receptive fields with on- and off-cells presenting direction-sensitive neural activities can be also applied for the directional sensitivity function denoted in equation (4) as another alternative.

\section{SIMULATION RESULTS}

The simulation uses the model represented by equations (2), (3) and (4) to observe the linkage pattern of edge-end pixels. Each parameter represented in the model equations influences the dynamics of the neural process. The parameters used in the simulation were obtained experimentally, which are given as: $a=0.1, b=0.05, c=0.5, e=$ $0.9, g=10^{5}$ and $\theta=10^{-3}$.

The simulations were performed in order to investigate whether the model equations would successfully accomplish the edge-linking process. The two signals applied to the neural model produce the results shown iteratively in Fig. 2 to Fig. 4.

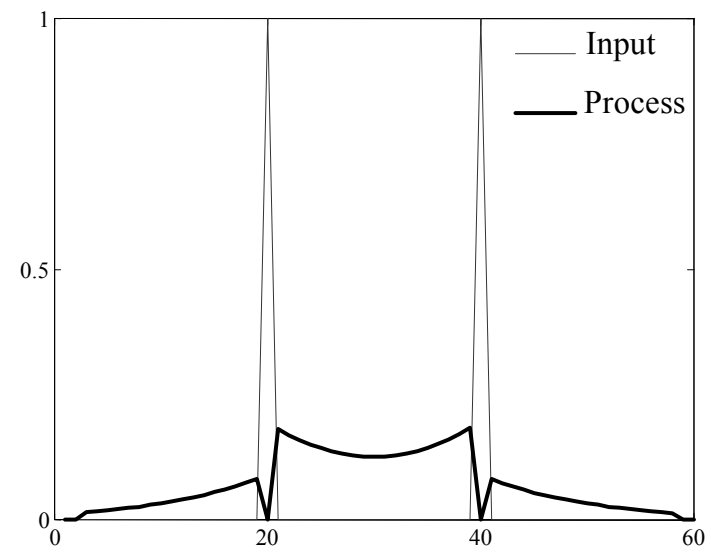

Fig. 2 - The linkage process starts in accordance with input signals of the two edge-end pixels.
The value of the input signal is set at 1 as the linkage technique applies to a binary image after applying other techniques that include edge detection and thresholding. Two edge-end pixels are considered for 1-D simulations through a series of 60 neurons.

The new value creation in accordance with the input signals of pixels is demonstrated by the simulation result in Fig. 2, which shows the initial pattern of linkage activation $z_{i}$ and the two inputs $I_{i}$. One of the iterations during the computer simulations shown in Fig. 3 demonstrates the behaviour of the linking process.

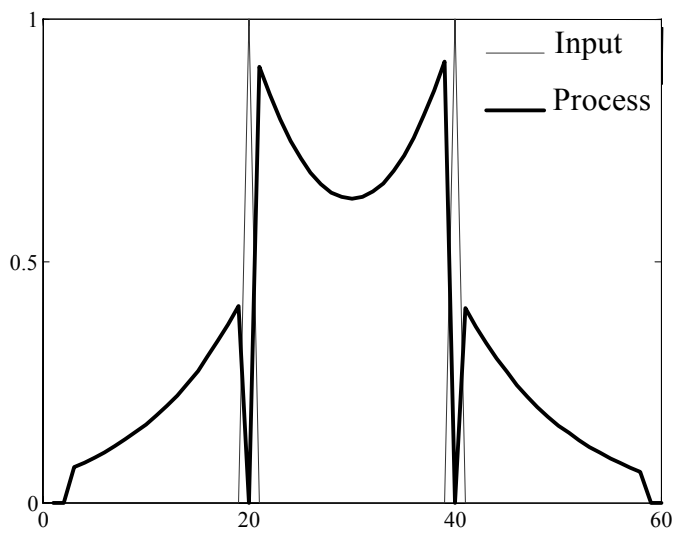

Fig. 3 - The linkage process starts in accordance with input signals of the two edge-end pixels.

There is an increase in activity where the process attempts to differentiate between the valid region and the spurious ones (Fig. 3 compared with Fig. 2). This activity slows down once the spurious ranges are identified and the linkage required between the edge-end pixels is established. The remainder of the process is for consolidation with the process tending toward the equilibrium status.

Fig. 4 shows the completion of the iteration process, which successfully performs the edgelinkage.

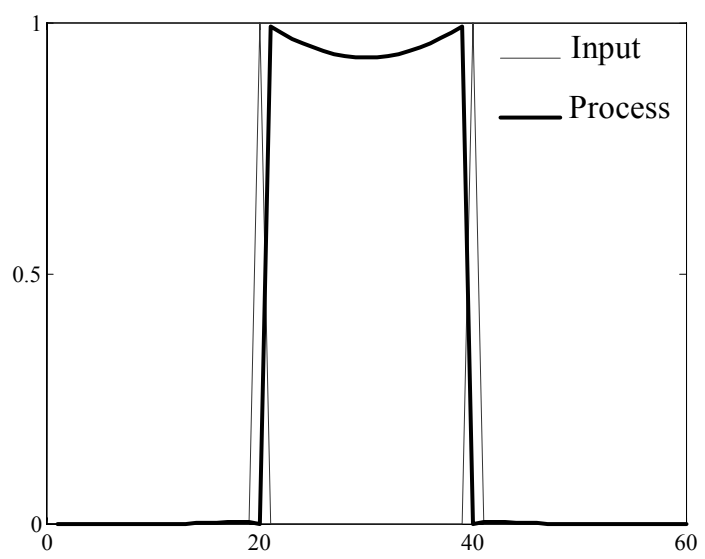

Fig. 4 - The linkage process for the edge-end pixels is completed to same magnitude of the input signal.

The neural response reaches equilibrium after 50 
runs in the simulations have been completed. The completed linking value of the two joined pixels does not exceed the value of the input magnitude, in this case 1 , after reaching equilibrium.

The two other spurious processing links shown in Fig. 3 are eliminated when the iterations achieve the conditions shown in Fig. 4 and the necessary linkage is retained. After obtaining the 2-D image displaying the new values for the links, the resultant image can easily be added programmatically to the initial image where linkage was yet to be established.

\section{CELL IMAGE ANALYSIS}

Negative-stained electron-microscopic image for SARS Coronavirus shown in Fig. 5 (a) was taken as a source image to be analysed as an example. The cell image analysis procedure is currently being implemented using the graphical G-language of LabVIEW $^{\circledR}$ program. Simply coupling a camera to an electron-microscope the source image acquired through a frame grabber will display on a graphical user interface created for the cell analysis using LabVIEW $^{\circledR}$.

The various steps with the image processing techniques programmatically control each resultant image displayed on the front panel. The steps used in this example include Gaussian smooth filtering, image buffer to the temporary memory, Sobel edge detection, thresholding, inverting binary image, morphological operations including the removal of small features and image border touching features, and thinning operation. IMAQ ${ }^{\circledR}$ functions introduced by National Instruments ${ }^{\circledR}$ facilitate the easier programming for user-friendly operation.

The application of edge detection and thresholding ultimately reveals discontinuities in cell boundaries as seen in Fig. 5 (b).

(a)

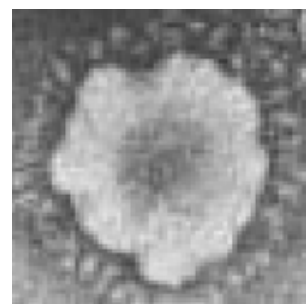

(c)
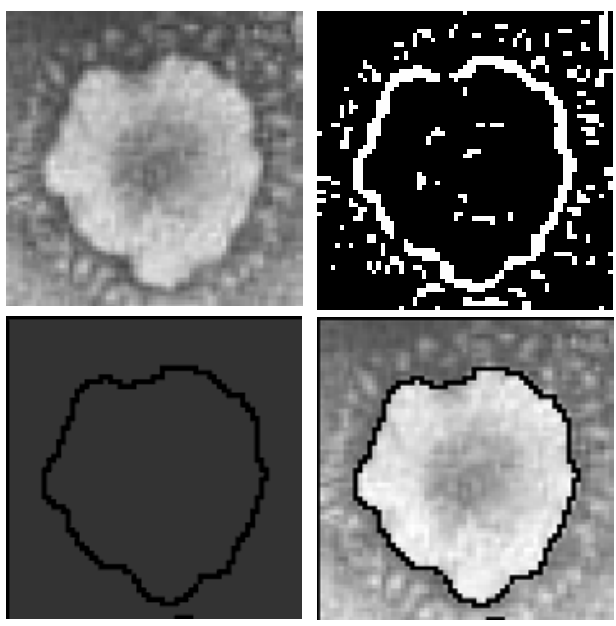

Fig. 5 - The application to the cell image analysis. this paper closes the tiny gaps without distorting the cell boundaries, as illustrated in Fig. 5 (c) after the thinning operation which produces a cell boundary of one pixel width. At this stage, the embedded virtual instrumentation platform labels the extracted cell object, after which the system automatically generates a detailed report with pre-programmed cell analysis tasks in a worksheet. The cell image is reconstituted without gaps in cell boundaries by superimposing the extracted cell object of Fig. 5 (c) on the source image of Fig. 5 (a), as shown in Fig. 5 (d). It is envisaged that benefits for clinicians will accrue in future if the images from each step and the report generated are transmitted live to clients' computers with embedded security features, situated remotely.

\section{CONCLUSION}

A novel approach to solve the edge gap problem experienced in medical image analysis has been successfully developed and tested. The simplicity of the proposed technique should make it an attractive pre-processing method for boundary extraction tasks in cell analysis, and indeed in any image analysis task.

\section{REFERENCES}

[1] G. Rizzo. P. Scifo. M.C. Gilardo. V. Bettinardi. F. Grassi. S. Cerutti and F. Fazio. Matching a computerized brain atlas to multimodal medical images, Neuroimage 6 (1997). p. 59-69.

[2] D. Hubel and T. Wiesel. Receptive fields, binocular interaction and functional architecture in the cat's visual cortex, Journal of Physiology 160 (1962). p. 106-154.

[3] D. Hubel and T. Wiesel. Receptive fields and functional architecture of monkey striate cortex, Journal of Physiology 195 (1968). p. 215-243.

[4] J. Krauskopf. Effect of retinal image stabilization on the appearance of heterochromatic targets, Journal of the Optical Society of America 53 (1963). p. 741-744.

[5] A.L. Yarbus. Eye Movements and Vision. Plenum Press. New York, 1967.

[6] E. Land. The retinex theory of color vision, Scientific American 237 (1977). p. 108-128.

[7] M. Piccolino. J. Neyton and H.M. Gerschenfeld. Decrease of gap junction permeability induced by dopamine and cyclic adenosine 3':5'monophosphate in horizontal cells of turtle retina, Journal of Neuroscience, 4 (1984). p. 2477-2788.

[8] H. Freeman. On the encoding of arbitrary geometric configurations, IEEE Trans. Elec. Computers 10 (1961). p. 260-268.

Applying the edge-linking technique proposed in 
[9] H. Freeman. Computer processing of linedrawing images, Computing Surveys 6 (1) (1974). p. 57-97.

[10] H. Freeman and L.S. Davis. A corner-finding algorithm for chain-coded curves, IEEE Trans. Computers 26 (1) (1977). p. 297-303.

[11] M. Pathegama. A neural mechanism and architecture for contour groupings and interactions via gated diffusion. Undergraduate Honours Thesis. University of South Australia, 2000.

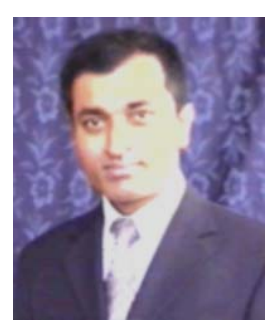

Mahinda Pathegama's education and career development are underscored by multidisciplinary engineering endeavours. He holds a Diploma in Electronic and Telecommunication engineering from the University of Moratuwa, Sri Lanka, and a Bachelor of Engineering degree with First Class Honours in Electrical and Mechatronic Engineering from the University of South Australia, Australia. He has been passionately interested in being able to model the human vision and the brain, particularly with the aid of artificial neural networks. His PhD research has utilised engineering skills crucial to medicine, applying artificial intelligence techniques to enhance diagnostic accuracy in medical practice. He has gained much recognition for his academic achievements by winning prestigious awards and prizes which have included the Sir William Goodman Electrical Engineering Prize, the Australian Postgraduate Award, the Chancellor's Award' and the Dean's Merit Award of the University of South Australia and the South Australian ETSA Utilities Prize. He is a Member of The Institution of Engineers, Australia (MIEAust), and a Member of the Institution of Electrical Engineers (MIEE), UK.

Özdemir Göl has well
recognised expertise in the
advanced application of
engineering techniques to
problems ranging from test
automation in manufacturing to
medical applications. He holds
the degrees of Master of

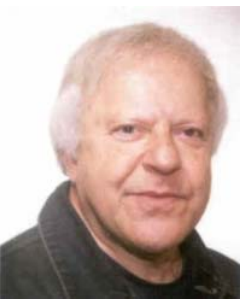

Engineering Science from the Istanbul Technical University, Turkey, Master of Engineering from the University of Melbourne, Australia, and PhD from the University of Adelaide, Australia; all in electrical engineering. $\mathrm{He}$ is currently Head of Electrical Engineering at the University of South Australia, where he also heads the Electrical Machines and Drives Research Group which he has founded. He has pioneered the use of virtual instrumentation techniques in Australia and has published a considerable number of journal and conference papers on the multidisciplinary application of VI techniques to the solution of a diverse range of problems. He conducts professional courses for practising scientists and engineers in addition to consulting for industry and research establishments. $\mathrm{He}$ is a Fellow of The Institution of Engineers, Australia, (FIEAust) and a Fellow of the Institution of Electrical Engineers, UK, (FIEE). 